Kinetic anomalies in addition-aggregation processes

This article has been downloaded from IOPscience. Please scroll down to see the full text article.

2003 J. Phys. A: Math. Gen. 364533

(http://iopscience.iop.org/0305-4470/36/16/304)

View the table of contents for this issue, or go to the journal homepage for more

Download details:

IP Address: 128.197.40.148

The article was downloaded on 06/12/2010 at 20:09

Please note that terms and conditions apply. 


\title{
Kinetic anomalies in addition-aggregation processes
}

\author{
M Mobilia, P L Krapivsky and S Redner \\ Center for BioDynamics, Center for Polymer Studies, and Department of Physics, \\ Boston University, Boston, MA 02215, USA \\ E-mail: mmobilia@buphy.bu.edu,paulk@bu.edu and redner@bu.edu
}

Received 20 January 2003, in final form 10 March 2003

Published 8 April 2003

Online at stacks.iop.org/JPhysA/36/4533

\begin{abstract}
We investigate irreversible aggregation in which monomer-monomer, monomer-cluster and cluster-cluster reactions occur with constant but distinct rates $K_{\mathrm{MM}}, K_{\mathrm{MC}}$ and $K_{\mathrm{CC}}$, respectively. The dynamics crucially depends on the ratio $\gamma=K_{\mathrm{CC}} / K_{\mathrm{MC}}$ and secondarily on $\epsilon=K_{\mathrm{MM}} / K_{\mathrm{MC}}$. For $\epsilon=0$ and $\gamma<2$, there is conventional scaling in the long-time limit, with a single mass scale that grows linearly in time. For $\gamma \geqslant 2$, there is unusual behaviour in which the concentration of clusters of mass $k, c_{k}$ decays as a stretched exponential in time within a boundary layer $k<k^{*} \propto t^{1-2 / \gamma}\left(k^{*} \propto \ln t\right.$ for $\left.\gamma=2\right)$, while $c_{k} \propto t^{-2}$ in the bulk region $k>k^{*}$. When $\epsilon>0$, analogous behaviours emerge for $\gamma<2$ and $\gamma \geqslant 2$.

PACS numbers: $02.50 .-\mathrm{r}, 05.40 .-\mathrm{a}, 68.43 . \mathrm{Jk}$
\end{abstract}

\section{Introduction}

In this work, we investigate a simple aggregation process in which three kinds of reactions occur with constant but distinct rates:

$$
\begin{aligned}
& A_{1}+A_{1} \stackrel{\epsilon}{\longrightarrow} A_{2} \\
& A_{1}+A_{j} \stackrel{1}{\longrightarrow} A_{j+1} \quad j \geqslant 2 \\
& A_{i}+A_{j} \stackrel{\gamma}{\longrightarrow} A_{i+j} \quad i, j \geqslant 2 .
\end{aligned}
$$

Equivalently, the reaction matrix $K_{i j}$ has the value $\epsilon$ in the upper left corner, the value 1 for all elements along the top and left edges of the matrix, and the value $\gamma$ in the rest of the matrix.

Despite its simplicity, this model exhibits rich dynamics in which both scaling and universality can be violated. The model was initially studied by Hendriks and Ernst [1] to describe polymerization in which addition processes (reactions involving monomers) occur more readily than aggregation (reactions between $i$ and $j$, with $i, j \geqslant 2$ ). They found evidence of unusual kinetic behaviour by an implicit solution for the cluster concentrations; see also [2] for related work. Our focus is on the opposite limit in which aggregation dominates over 
addition. By explicit solutions of the rate equations, we elucidate the full range of behaviours for this system.

From a general perspective, the origin of the anomalous behaviour in addition/aggregation stems from the fact that the simplest case of aggregation with a constant reaction rate is actually a marginal system. As discussed by van Dongen and Ernst [3], aggregation can be broadly categorized by whether reactions among large clusters dominate (type I systems, in their nomenclature) or whether reactions between large and small clusters dominate (type III systems). The former generically leads to monotonically decaying cluster mass distributions, while the latter gives peaked distributions. The constant reaction rate system (type II system) is marginal by being at the boundary between these two behaviours.

Because of this marginality, the kinetics of constant-kernel aggregation is sensitive to small perturbations in the reaction rates. Previously-studied examples of this feature include aggregation involving two distinct monomeric units, so that the reaction rates between two even masses, two odd masses, or an even and an odd mass are naturally distinct. Here nonuniversal and non-scaling behaviour arises as a function of these rates $[4,5]$. Another example is aggregation with the rate $K_{i j}=2-q^{i}-q^{j}$ with $0<q<1$ [6]. Although this reduces to a constant-kernel system for $i, j \rightarrow \infty$, the $q$-dependent terms lead to unusual kinetics. The model studied here is in the spirit of the first example, except that we perturb only an infinitesimal fraction of the reaction rates in a constant-kernel system. It is striking that such a small change in the reaction rates has such a profound influence on the kinetics.

In the next two sections, we focus on the special case of 'sterile' monomers, where monomer-monomer reactions do not occur. In section 2, we first determine the monomer and dimer concentrations and show that different behaviours arise for $\gamma \geqslant 2$ and $\gamma<2$. Then in section 3, we derive asymptotic results for the cluster concentrations. We then study, in section 4, these same three cases when monomer-monomer reactions can also occur. Section 5 gives a summary as well as a discussion of the equivalence between the model and a diffusion-controlled process in which monomers have different diffusivity than other clusters.

\section{Sterile monomers}

When monomers do not interact among themselves $\left(K_{11}=0\right)$, we shall show that the system exhibits three distinct kinetic regimes, with unusual time dependences and breakdown of conventional scaling in two of these cases.

The rate equation for the density $c_{k}(t)$ is [7]

$$
\dot{c}_{k}(t)=\frac{1}{2} \sum_{i+j=k} K_{i j} c_{i}(t) c_{j}(t)-c_{k}(t) \sum_{j \geqslant 1} K_{k j} c_{j}(t)
$$

where the overdot denotes time derivative. The first (gain) term accounts for the formation of clusters of mass $k$ ( $k$-mers) as a result of the bimolecular aggregation of $i$-mers with $j$-mers, with $i+j=k$. The second (loss) term accounts for the aggregation of the $k$-mers with any $j$-mer, thus leading to a decrease in the $k$-mer concentration. For the processes given in (1) and with the additional constraint of $K_{11}=\epsilon=0$, the explicit rate equations are

$$
\begin{aligned}
\dot{c}_{1} & =-c_{1} R \\
\dot{c}_{2} & =-c_{2}\left[c_{1}+\gamma R\right] \\
\dot{c}_{k} & =c_{1} c_{k-1}+\frac{\gamma}{2} \sum_{\substack{i+j=k \\
i, j \geqslant 2}} c_{i} c_{j}-c_{k}\left(c_{1}+\gamma R\right) \quad k>2
\end{aligned}
$$


where we drop the time argument for compactness and introduce the shorthand $R \equiv \sum_{k \geqslant 2} c_{k}$ for the concentration of reactive clusters (those with mass $\geqslant 2$ ). Note also that there is no production of monomers and dimers in the reaction; they can only disappear.

The governing equation for $R$ is obtained by summing equations (3) to give $\dot{R}=-\frac{\gamma}{2} R^{2}$, whose solution is

$$
R=R(0) \tau^{-1} \quad \tau \equiv 1+\frac{1}{2} \gamma R(0) t .
$$

With the explicit expression for $R$, we integrate the equation for monomers to give

$$
c_{1}=c_{1}(0) \tau^{-2 / \gamma}
$$

The crucial point is that the value of $\gamma$ controls which of $R$ or $c_{1}$ decays more rapidly. For $\gamma<2$, monomers are asymptotically irrelevant and conventional constant-kernel aggregation kinetics arises. For $\gamma \geqslant 2$, however, the large disparity in the reaction rates $K_{1 j}$ and $K_{i j}$ leads to a long-lived residue of monomers and these profoundly affect the asymptotic kinetics.

To see this explicitly, we solve for the density of dimers to find

$$
c_{2}=c_{2}(0) \tau^{-2} \mathrm{e}^{-\lambda}
$$

where we define $\lambda=\int_{0}^{t} \mathrm{~d} t^{\prime} c_{1}\left(t^{\prime}\right)$. Using equation (5) we express $\lambda$ in terms of $\tau$ :

$$
\lambda \equiv \lambda(\tau)= \begin{cases}2 r \frac{\tau^{1-2 / \gamma}-1}{\gamma-2} & \gamma \neq 2 \\ r \ln \tau & \gamma=2\end{cases}
$$

where we further define $r=c_{1}(0) / R(0)$.

Depending on the value of $\gamma$ we obtain three distinct long-time behaviours:

- For $0<\gamma<2, \lambda \rightarrow \frac{2 r}{2-\gamma} \rightarrow$ const as $t \rightarrow \infty$. Thus the dimer concentration has a universal $t^{-2}$ asymptotic decay; only the amplitude of the decay depends on the initial state.

- For $\gamma>2$, the dimer density decays as a stretched exponential $\mathrm{e}^{-\lambda}$ times a power-law prefactor.

- For $\gamma=2$, the dimer concentration asymptotically decays as $t^{-(2+r)}$, where the exponent depends on the initial concentration ratio $r=c_{1}(0) / R(0)$.

\section{Asymptotic mass distribution}

To determine the asymptotic cluster concentrations $c_{k}$, we rewrite the rate equations (3) in the form

$$
\dot{c}_{k}+\alpha c_{k}=\alpha_{k}
$$

where

$$
\alpha \equiv c_{1}+\gamma R \quad \alpha_{k} \equiv c_{1} c_{k-1}+\frac{\gamma}{2} \sum_{\substack{i+j=k \\ i, j \geqslant 2}} c_{i} c_{j} .
$$

Due to the recursive nature of equation (8) we immediately obtain the formal solution for $c_{k}$ :

$$
c_{k}(t)=\mathcal{E}(t)\left\{c_{k}(0)+\int_{0}^{t} \mathrm{~d} t^{\prime} \frac{\alpha_{k}\left(t^{\prime}\right)}{\mathcal{E}\left(t^{\prime}\right)}\right\}
$$

with $\mathcal{E}(t)=\exp \left[-\int_{0}^{t} \mathrm{~d} t^{\prime} \alpha\left(t^{\prime}\right)\right]=\tau^{-2} \mathrm{e}^{-\lambda}$. Therefore, once we know $c_{1}, \ldots, c_{k-1}$, we can compute $\alpha_{k}$ and then determine $c_{k}$. 
Before proceeding to general $k$, it is instructive to compute $c_{3}$. From equation (10), and using the previous results for $c_{1}$ and $c_{2}$, we obtain

$$
c_{3}=\left[c_{2}(0) \lambda+c_{3}(0)\right] \tau^{-2} \mathrm{e}^{-\lambda} .
$$

As in the case of dimers, there are three distinct asymptotic behaviours for the trimer concentration that are best expressed in terms of $c_{3} / c_{2}$. We obtain

$$
\frac{c_{3}}{c_{2}} \propto \begin{cases}t^{1-2 / \gamma} & \gamma>2 \\ \ln t & \gamma=2 \\ 1 & \gamma<2\end{cases}
$$

that is, $c_{3} / c_{2} \propto \lambda$ for any value of $\gamma$ (see equation (7)). While the exact expressions for the concentrations $c_{k}$ become unwieldy as $k$ grows, the asymptotic expressions turn out to be simple: $c_{3} / c_{2} \rightarrow \lambda, c_{4} / c_{2} \rightarrow \lambda^{2} / 2$, etc. We will confirm this hypothesis by induction in the following subsections.

\section{1. $A: \gamma \geqslant 2$}

When $\gamma$ is strictly greater than 2 , we easily find that $c_{k} / c_{2} \propto \lambda^{k-2}$ as $t \rightarrow \infty$ by explicit calculation for small $k$. We therefore seek an asymptotic solution for $c_{k}$ of the form

$$
\frac{c_{k}}{c_{2}}=\beta_{k} \lambda^{k-2}+\cdots
$$

where ... denotes subdominant terms in the limit $t \rightarrow \infty$ and the amplitude $\beta_{k}$ will be determined below.

For concreteness and simplicity, let us consider the bi-disperse initial condition in which only $c_{1}(0)$ and $c_{2}(0)$ are non-zero while $c_{k}(0)=0$ for all $k>2$. To extract the asymptotics of $c_{k}$ from equation (10), only the second term on the right-hand side is needed. Let us suppose that equation (13) holds for $c_{2}, \ldots, c_{k}$. To leading order, we have $\alpha_{k+1}=c_{1} c_{k}$, and substituting $c_{k}=c_{2} \beta_{k} \lambda^{k-2}$ into equation (10), we obtain

$$
c_{k+1}=\mathcal{E}(t) \int_{0}^{t} \mathrm{~d} t^{\prime} \frac{c_{1}\left(t^{\prime}\right) c_{k}\left(t^{\prime}\right)}{\mathcal{E}\left(t^{\prime}\right)}=c_{2} \frac{\beta_{k}}{k-1} \lambda^{k-1}
$$

where to obtain the second equality we use $\mathcal{E}(t)=\tau^{-2} \mathrm{e}^{-\lambda}$. Thus $\beta_{k}=1 /(k-2)$ ! and we arrive at the remarkably simple asymptotic solution

$$
c_{k+2}=c_{2} \frac{\lambda^{k}}{k !} \quad c_{2}=c_{2}(0) \tau^{-2} \mathrm{e}^{-\lambda}
$$

with $\tau$ and $\lambda$ given by equations (4) and (7). Explicitly, we have

$$
c_{k+2} \sim \frac{1}{k !} t^{-2+k(1-2 / \gamma)} \exp \left(- \text { const } \times t^{1-2 / \gamma}\right) .
$$

Thus $c_{k}$ has a very different asymptotic behaviour than in the constant-kernel system [7]. As discussed previously, this anomaly arises because monomers are very long-lived and these then strongly influence the long-time kinetics.

One important caveat, however, is that the derivation of equation (15) applies only for finite $k$. For sufficiently large $k$ the neglected subdominant terms accumulate to provide a relevant contribution. The simplest way to see that equation (15) cannot hold over entire mass range is to use this equation to compute the total cluster density. This gives $\sum_{k \geqslant 2} c_{k}=c_{2} \mathrm{e}^{\lambda}=c_{2}(0) \tau^{-2}$, in contrast to the correct result $R(0) \tau^{-1}$ given in equation (4).

We therefore conclude that the mass distribution naturally divides into a small-mass boundary layer, $k \leqslant k^{*}$, that contains an asymptotically negligible fraction of the total mass, 
and a remaining bulk region. The extent of the boundary layer may be determined as the maximum of $c_{k}$ in equation (15) and gives $k^{*}=\lambda \propto t^{1-2 / \gamma}$. Within this boundary layer, the mass distribution grows rapidly with mass, but this region contains only an asymptotically negligible fraction of the total mass. On the other hand, most of the mass, which exhibits conventional scaling behaviour is contained in the bulk region.

In the marginal case of $\gamma=2$, the asymptotic solution of equation (15) is still valid. Since now $\lambda=r \ln \tau$, the density of dimers decays algebraically in time as $c_{2}=c_{2}(0) \tau^{-(2+r)}$, and the width of the boundary layer grows logarithmically $k^{*}=\lambda=r \ln \tau$. The following main part of the mass distribution is again characterized by conventional scaling behaviour (see equation (19)). A justification of this picture is given in the appendix. Thus for $\gamma \geqslant 2$, the mass distribution exhibits two growing scales and conventional single-mass scaling is violated.

\section{2. $B: \gamma<2$}

By solving $c_{2}, c_{3}, \ldots$ exactly, we are led to the hypothesis that for $k \geqslant 2$

$$
c_{k} \propto \tau^{-2} \text {. }
$$

This indeed can be directly checked by induction. Moreover, in the scaling limit,

$$
k \rightarrow \infty \quad t \rightarrow \infty \quad \frac{k}{t}=\text { finite }
$$

the mass distribution admits the conventional scaling form

$$
c_{k}=\frac{4}{\gamma^{2} t^{2}} \mathrm{e}^{-2 k /(\gamma t)} .
$$

Summing $\sum c_{k}$ we indeed recover $R=2 /(\gamma t)$, while the next moment $\sum k c_{k}$ equals one (based on the total mass set equal to one initially). Equation (19) also describes the scaling portion of the mass distribution when $\gamma \geqslant 2$.

The origin of the conventional scaling behaviour for the case $\gamma<2$ is simple. For $\gamma<2$ monomers disappear quickly, since $c_{1} \propto t^{-2 / \gamma}$, and their asymptotic influence is negligible. Thus the reaction effectively reduces to a constant-kernel system that begins with dimers. The monomers do influence the small-mass behaviour, namely $c_{k}=A_{k} t^{-2}$ with mass-dependent amplitudes $A_{k}$; e.g., $A_{2}=c_{2}(0)[\gamma R(0) / 2]^{-2} \mathrm{e}^{-\lambda}$ and $A_{3}=A_{2}\left[\lambda+c_{3}(0) / c_{2}(0)\right]$. However, as $k$ grows the amplitude $A_{k}$ approaches the constant value $4 \gamma^{-2}$.

The existence of scaling can be proved rigorously, e.g., by the generating function approach given in the appendix. A simpler approach is to merely assume that scaling holds and check its consistency a posteriori. In the continuum limit, we have checked the correctness of equation (19), which is the conventional scaling form for constant-kernel aggregation [7].

\section{Reactive monomers}

We now assume that monomer-monomer reactions do occur: $K_{11} \equiv \epsilon>0$. This situation has already been studied by Hendriks and Ernst [3]. The rate equations for monomers and dimers now read

$$
\begin{aligned}
& \dot{c}_{1}=-c_{1}\left(\epsilon c_{1}+R\right) \\
& \dot{c}_{2}=\frac{\epsilon}{2} c_{1}^{2}-c_{2}\left(c_{1}+\gamma R\right)
\end{aligned}
$$

while the rate equations for clusters with $k>2$ are the same as in equation (3). Correspondingly, the density of reactive clusters $R$ evolves according to

$$
\dot{R}=\frac{1}{2} \epsilon c_{1}^{2}-\frac{1}{2} \gamma R^{2} \text {. }
$$


It does not seem possible to solve the coupled non-linear equations (20) and (22) for $c_{1}$ and $R$ explicitly. If we treat $c_{1}$ as a function of $R$, however, we can reduce equations (20) and (22) to a single differential equation that can be solved to establish a functional relation between $c_{1}$ and $R$ [1]. From this relation, we can deduce that different behaviours emerge depending on whether $\gamma<2$ or $\gamma \geqslant 2$.

A simpler and more fruitful way to proceed is to seek the asymptotic behaviour of $c_{1}$ and $R$ without explicitly solving the governing equations. We anticipate that there are three possible asymptotic behaviours: (i) $c_{1} \ll R$, (ii) $c_{1} \propto R$ and (iii) $c_{1} \gg R$. Substituting each of these scenarios into equations (20) and (22) shows that the latter case is impossible. Let us now analyse the first two possibilities. When $c_{1} \ll R$, equation (22) gives $R \simeq 2 /(\gamma t)$, and then equation (20) yields $c_{1} \propto t^{-2 / \gamma}$. Thus the relation $c_{1} \ll R$ holds when $\gamma<2$. In the complementary regime of $\gamma>2$, we find that $c_{1} \propto R$ is consistent; furthermore, equations (20) and (22) now give $R \propto c_{1} \propto t^{-1}$.

We now analyse these two cases in more detail.

\section{1. $A: \gamma \geqslant 2$}

When $\gamma$ is strictly greater than 2, we substitute the ansatz

$$
R \simeq A t^{-1} \quad c_{1} \simeq B t^{-1}
$$

into equations (20) and (22) to get a quadratic equation for the amplitude $A$. The physical requirement that $A$ and $B$ are both positive fixes the solution to be

$$
A=\frac{\epsilon-1+\sqrt{\epsilon(\epsilon+\gamma-2)}}{\epsilon \gamma-1} \quad B=\frac{1-A}{\epsilon} .
$$

The singularity at $\epsilon=\gamma^{-1}$ is only apparent and may be resolved by applying the l'Hospital's rule, to give $A=\gamma /[2(\gamma-1)]$ in this case.

From the formal solution (10) we can check by induction that for finite $k$,

$$
c_{k} \simeq B_{k} t^{-1} \text {. }
$$

The amplitude $B_{k}$ is found by substituting the ansatz (25) into (8), (9) to give the recursion

$$
(\mu-1) B_{k}=B B_{k-1}+\frac{\gamma}{2} \sum_{\substack{i+j=k \\ i, j \geqslant 2}} B_{i} B_{j}
$$

for $k>2$, where we define

$$
\mu=\gamma A+B=1+\sqrt{1+(\gamma-2) / \epsilon} .
$$

Note also that $(\mu-1) B_{2}=\epsilon B^{2} / 2$. To solve the recursion (26) we introduce the generating function $\mathcal{B}(z)=\sum_{k \geqslant 2} B_{k} z^{k}$ to reduce (26) to

$$
\mathcal{B}(z)=\frac{\mu-1-B z}{\gamma}\left\{1-\sqrt{1-\epsilon \gamma\left(\frac{B z}{\mu-1-B z}\right)^{2}}\right\} .
$$

When $k \gg 1$ but still within the boundary layer, the asymptotic behaviour of $\mathcal{B}(z)$ leads to the amplitude

$$
B_{k} \simeq C k^{-3 / 2} \beta^{k}
$$

with

$$
\beta=B \frac{1+\sqrt{\epsilon \gamma}}{\mu-1} \quad C=\frac{\mu-1}{\gamma \sqrt{2 \pi}} \sqrt{\frac{\sqrt{\epsilon \gamma}}{1+\sqrt{\epsilon \gamma}}} .
$$


One can verify that $\beta<1$ for all $\gamma>2$ and $\epsilon>0$; therefore, apart from a power-law prefactor, the amplitude $B_{k}$ decreases exponentially with $k$.

As in the previous section, the results (25) and (28) are valid only within the boundary layer. Thus the total density of clusters within the boundary layer is asymptotically equal to $A^{\prime} t^{-1}$, where $A^{\prime}=\sum_{k \geqslant 2} B_{k}=\mathcal{B}(z=1)$, or

$$
A^{\prime}=\frac{\mu-1-B-\sqrt{(\mu-1-B)^{2}-\epsilon \gamma B^{2}}}{\gamma} .
$$

Since the total density of clusters scales as $A t^{-1}$, only a fraction $A^{\prime} / A$ of clusters lies within the boundary layer. Using equation (24) and (30), we find $A-A^{\prime}=2 / \gamma$; this implies that the density of clusters in the bulk decays as $2 /(\gamma t)$. The same holds for $\gamma \leqslant 2$, although in this case the density of clusters in the boundary layer is asymptotically negligible.

To determine the asymptotic behaviour in the bulk $(k \rightarrow \infty$ and $t \rightarrow \infty)$, we simplify the rate equations by neglecting the subdominant terms $c_{1}\left(c_{k-1}-c_{k}\right)$ on the right-hand side of equation (3). Further, the sum in (3) has three contributions. When $i$ lies within the boundary layer, we can replace $\sum_{i} c_{i} c_{k-i}$ by $c_{k} \sum_{i} B_{i} t^{-1}=c_{k} A^{\prime} t^{-1}$; a similar contribution arises when $j$ lies within the boundary layer. In the bulk we replace the sum by the integral. Thus

$$
\frac{\gamma}{2} \sum_{i+j=k} c_{i} c_{j} \rightarrow \frac{\gamma}{2} \int \mathrm{d} i c_{i} c_{k-i}+\frac{\gamma A^{\prime}}{t} c_{k} .
$$

Combining all terms and using the identity $\gamma\left(A-A^{\prime}\right)=2$ we finally convert equation (3) to

$$
\dot{c}_{k}+\frac{2}{t} c_{k}=\frac{\gamma}{2} \int \mathrm{d} i c_{i} c_{k-i}
$$

This equation appears in the standard constant-kernel aggregation and its solution, satisfying the aforementioned conservation laws $\int \mathrm{d} k c_{k}=2 / \gamma$ and $\int \mathrm{d} k k c_{k}=1$, is given by equation (19).

By matching the mass distribution in the boundary layer $\left(c_{k} \propto t^{-1} \beta^{k}\right)$ and in the bulk $\left(c_{k} \propto t^{-2}\right)$, we estimate the width of the boundary layer as

$$
k^{*} \approx \frac{\ln t}{\ln (1 / \beta)}
$$

with $\beta$ given by equation (29). This is a much slower growth than in the case of sterile monomers where $k^{*} \propto t^{1-2 / \gamma}$.

When $\gamma=2$, we again expect marginal behaviour. Proceeding as in the case of $\gamma>2$, we find

$$
R \simeq t^{-1} \quad c_{1} \simeq t^{-1}(\epsilon \ln t)^{-1} .
$$

From the formal solution (10) we can check by induction that

$$
c_{k} \simeq B_{k} t^{-1}(\epsilon \ln t)^{-k} \text {. }
$$

For $k>2$, the amplitude $B_{k}$ is found from the recursion (26) with $\mu=2, B=1$ and $B_{2}=\epsilon / 2$. The generating function and the coefficients $B_{k}$ and $C$ are given by taking the corresponding formulae in the $\gamma>2$ case and setting $\gamma=2$.

This behaviour holds in a boundary layer whose width now grows as

$$
k^{*} \approx \frac{\ln t}{\ln (\Lambda \ln t)} \quad \Lambda=\frac{\epsilon}{1+\sqrt{2 \epsilon}}
$$

while in the bulk we recover the ordinary scaling mass distribution $c_{k}=t^{-2} \mathrm{e}^{-k / t}$. 


\section{2. $B: \gamma<2$}

We already argued that $R \simeq 2 /(\gamma t)$ and $c_{1} \propto t^{-2 / \gamma}$ for $\gamma<2$. Solving then for dimers we obtain

$$
c_{2} \propto \begin{cases}t^{-2} & 0<\gamma<4 / 3 \\ t^{1-4 / \gamma} & 4 / 3<\gamma<2 .\end{cases}
$$

Generally, we find

$$
c_{k} \propto \begin{cases}t^{-2} & 0<\gamma<\frac{2 k}{k+1} \\ t^{-2} \ln t & \gamma=\frac{2 k}{k+1} \\ t^{k-1-2 k / \gamma} & \frac{2 k}{k+1}<\gamma<2 .\end{cases}
$$

Thus for every $\gamma<2$, small-mass clusters have abnormal kinetics: only monomers for $\gamma<4 / 3$, monomers and dimers for $4 / 3 \leqslant \gamma<3 / 2$, monomers, dimers and trimers for $3 / 2 \leqslant \gamma<8 / 5$, etc. The remaining clusters decay as $t^{-2}$, and conventional scaling describes the cluster-mass distribution.

\section{Summary and discussion}

For irreversible aggregation with distinct monomer-monomer $\left(K_{\mathrm{MM}}\right)$, monomer-cluster $\left(K_{\mathrm{MC}}\right)$ and cluster-cluster $\left(K_{\mathrm{CC}}\right)$ reaction rates, the dynamics depend crucially on the ratio $\gamma=K_{\mathrm{CC}} / K_{\mathrm{MC}}$, while $\epsilon=K_{\mathrm{MM}} / K_{\mathrm{MC}}$ plays a lesser role-all that matters is whether $\epsilon=0$ or $\epsilon>0$. For $\epsilon=0$ and $\gamma<2$, there is conventional scaling with a single mass scale growing linearly with time. For $\gamma \geqslant 2$, there are two scales: the boundary layer $k<k^{*} \propto t^{1-2 / \gamma}$ where the mass distribution has an unusual Poisson form, and the bulk region $k>k^{*}$ where conventional scaling holds. When $\epsilon>0$ and $\gamma<2$, there is conventional scaling, except that light clusters have abnormal kinetics-monomers for $\gamma<4 / 3$; monomers and dimers for $4 / 3 \leqslant \gamma<3 / 2$; monomers, dimers and trimers for $3 / 2 \leqslant \gamma<8 / 5$, etc. When $\epsilon>0$ and $\gamma \geqslant 2$, the behaviour in the boundary layer is very different from that when $\epsilon=0$. In particular, the mass distribution decays with mass while for $\epsilon=0$ the mass distribution is peaked.

A possibly useful reformulation of this model is to clustering on surfaces. Consider a two-dimensional substrate with diffusing single-layer islands that aggregate whenever they meet. In the diffusion-controlled limit, the reaction rate of an island of radius $R$ and diffusivity $D$ is proportional to $D \ln R$ [8]. To a good approximation, we can ignore the island radius and think of point-like islands that always occupy a single-lattice site. When such a cluster hops onto already occupied site, two clusters immediately coalesce into a single cluster.

If monomers hop with rate $D$ while all heavier clusters hop with the same unit rate, then the corresponding reaction rates for point-like islands are $K_{11}=2 D, K_{1 j}=1+D, K_{i j}=2$. As a result

$$
\epsilon=\frac{K_{11}}{K_{1 j}}=\frac{2 D}{1+D} \quad \gamma=\frac{K_{i j}}{K_{1 j}}=\frac{2}{1+D} .
$$

For immobile monomers, $D=0$, we have $\epsilon=0$ and $\gamma=2$. In this case, the cluster mass distribution is

$$
c_{k+2}=c_{2}(0) \tau^{-(2+r)} \frac{\lambda^{k}}{k !}
$$

within a boundary layer that grows logarithmically with time $k^{*}=r \ln (1+R(0) t)$, where $r=c_{1}(0) / R(0)$. In the bulk, ordinary scaling holds in which $c_{k}=t^{-2} \mathrm{e}^{-k / t}$. 
When $D>0, \gamma$ is always less than 2 . Therefore,

$$
R=\frac{1+D}{t} \quad c_{1} \propto t^{-1-D} \text {. }
$$

The decay rate for clusters of mass $k>1$ is also simple:

$$
c_{k} \propto \begin{cases}t^{-2} & D>1 / k \\ t^{-2} \ln t & D=1 / k \\ t^{-1-k D} & D<1 / k .\end{cases}
$$

\section{Acknowledgments}

MM thanks the Swiss NSF for financial support under the fellowship 81EL-68473. PLK and SR thank NSF grant DMR9978902 for financial support of this research.

\section{Appendix A. Generating function approach}

In the case of sterile monomers, we can employ the generating function approach as an alternative to a direct solution. The generating function

$$
G(z, t) \equiv \sum_{k \geqslant 2} z^{k} c_{k}(t)
$$

recasts an infinite set of rate equations into a single differential equation

$$
\frac{\partial G}{\partial t}=\frac{\gamma}{2} G^{2}+\left(z c_{1}-\alpha\right) G
$$

This is a Bernoulli equation that is readily solved in terms of $G^{-1}$. The solution is

$$
G^{-1}=\frac{\tau^{2} \mathrm{e}^{(1-z) \lambda}}{G(z, 0)}-\int_{1}^{\tau} \frac{\mathrm{d} \tau^{\prime}}{R(0)}\left(\frac{\tau}{\tau^{\prime}}\right)^{2} \mathrm{e}^{(1-z)\left(\lambda-\lambda^{\prime}\right)}
$$

where $\lambda=\lambda(\tau)$ and $\lambda^{\prime}=\lambda\left(\tau^{\prime}\right)$. For the bi-disperse initial condition, $c_{k}(0)=c_{1}(0) \delta_{k, 1}+$ $c_{2}(0) \delta_{k, 2}$, we have $G(z, 0)=c_{2}(0) z^{2}$ and $R(0)=c_{2}(0)$. Using these relations together with $c_{2}=c_{2}(0) \tau^{-2} \mathrm{e}^{-\lambda}$ we rewrite $(\mathrm{A} 3)$ as

$$
G^{-1}=\frac{\mathrm{e}^{-z \lambda}}{c_{2} z^{2}}-\frac{\mathrm{e}^{-z \lambda}}{c_{2}} \int_{1}^{\tau} \frac{\mathrm{d} \tau^{\prime}}{\left(\tau^{\prime}\right)^{2}} \mathrm{e}^{-(1-z) \lambda^{\prime}} .
$$

We now consider in detail the marginal case of $\gamma=2$ and justify the two-scale structure of the mass distribution. When $\gamma=2$, we have $\lambda=r \ln \tau$ and (A4) becomes

$$
G(z, t)=\frac{c_{2}(t) z^{2} \mathrm{e}^{\lambda z}\left(1-\frac{r}{1+r} z\right)}{1-\frac{r}{1+r} z-\frac{z^{2}}{1+r}\left[1-\tau^{-1-r(1-z)}\right]} .
$$

The term $\tau^{-1-r(1-z)}$ in the denominator can be ignored within the boundary layer. Therefore, the denominator becomes $(1-z)\left[1+(1+r)^{-1} z\right]$, and the generating function simplifies to

$$
G(z, t)=c_{2}(t) z^{2} \mathrm{e}^{\lambda z} \frac{1}{2+r}\left[\frac{1}{1-z}+\frac{1+r}{1+\frac{z}{1+r}}\right] .
$$

To extract the mass distribution, we expand $G(z, t)$ in a Taylor series in $z$. This gives

$$
\frac{c_{k+2}}{c_{2}}=\sum_{n=0}^{k} \frac{\lambda^{n}}{n !}\left[\frac{1}{2+r}+\frac{1+r}{2+r}\left(-\frac{1}{1+r}\right)^{k-n}\right] .
$$


Thus apart from the leading contribution that equals $\lambda^{k} / k$ !, in agreement with our previous result in equation (15), we find all the correction terms; e.g., the leading correction is $(1+r)^{-1} \lambda^{k-2} /(k-2) !$.

When both the quadratic and the transcendental terms in the denominator balance each other, the generating function accounts for the main part of the mass distribution. The both terms are comparable when $1-z \propto \tau^{-1}$. Writing $1-z=\zeta / \tau$ and taking $z \rightarrow 1, \tau \rightarrow \infty$ limit, with $\zeta$ kept finite, we simplify the generating function

$$
G=\frac{c_{2}(0)}{\tau} \frac{1}{1+(2+r) \zeta} .
$$

This form of the generating function implies that

$$
c_{k}=\frac{c_{2}(0)}{(2+r) \tau^{2}} \exp \left[-\frac{1}{2+r} \frac{k}{\tau}\right] .
$$

Since $\gamma=2$ and $(2+r) R(0)=2 c_{2}(0)+c_{1}(0)=1$ (for the bi-disperse initial conditions $R(0)=c_{2}(0)$ and the mass density is always set equal to one), the above scaling form reduces to the anticipated scaling form of equation (19).

\section{References}

[1] Hendriks E M and Ernst M H 1984 J. Colloid Interface Sci. 97176

[2] Samsel R W and Perelson A S 1982 Biophys. J. 37493

Perelson A S and Samsel R W 1984 Kinetics of Aggregation and Gelation ed F Family and D P Landau (Amsterdam: North-Holland)

[3] van Dongen P G J and Ernst M H 1983 J. Phys. A: Math. Gen. 16 L327 van Dongen P G J and Ernst M H 1985 Phys. Rev. Lett. 541396

[4] Leyvraz F and Redner S 1986 Phys. Rev. Lett. 57163 Leyvraz F and Redner S 1987 Phys. Rev. A 364033

[5] Calogero F and Leyvraz F 2000 J. Phys. A: Math. Gen. 335619

[6] Calogero F and Leyvraz F 1999 J. Phys. A: Math. Gen. 327697

[7] See, e.g. Friedlander S K 1977 Smoke, Dust and Haze: Fundamentals of Aerosol Behaviour (New York: Wiley) Ernst M H 1985 Fundamental Problems in Statistical Physics VI ed E G D Cohen (New York: Elsevier)

[8] Chandrasekhar S 1943 Rev. Mod. Phys. 151 